\title{
Especificación de un modelo para el estudio de las redes colaborativas en una universidad productora de conocimiento ${ }^{1}$
}

\author{
Specification of a model for the study of collaborative networks in a knowledge \\ producing university
}

Recibido: 19 de abril de 2017 - Revisado: 28 de agosto de 2017 - Aceptado: 12 de febrero de 2018.

\author{
Wilfrido I. Aldana-Balderas ${ }^{2}$ \\ Francisco Espinoza-Morales ${ }^{3}$ \\ Jorge Hernández-Valdés ${ }^{4}$ \\ Cruz García-Lirios ${ }^{5}$
}

\begin{abstract}
Resumen
Las redes de conocimiento y la colaboración organizacional son reflejo de una cultura del éxito, liderazgo transformacional y clima de relaciones en torno a las que se gestan relaciones de confianza, apoyo, innovación y metas. Se trata de organizaciones bidireccionales y horizontales con equidad y solidaridad. El objetivo del presente trabajo es establecer las correlaciones entre los factores. Se llevó a cabo un estudio no experimental, transversal y exploratorio con una selección de 300 administrativos, estudiantes y docentes de una universidad pública del centro de México. A partir de un modelo estructural, los resultados muestran que existe una relación de dependencia entre el clima de metas y la colaboración. A partir de estos hallazgos se advierten líneas de investigación relativas a la confianza como determinante de las redes de conocimiento y la colaboración organizacional.
\end{abstract}

\section{Palabras clave}

Cultura laboral, institucionalismo, liderazgo, red de conocimiento, colaboración.

\begin{abstract}
knowledge networks and organizational collaboration reflect a culture of success, transformational leadership and a climate of relationships around which relationships of trust, support and innovation are generated. These are bi-directional and horizontal organizations with equity and solidarity. The objective of the present study is to establish the correlations between the factors, a non-experimental, transversal and exploratory study was carried out with a selection of 300 administrative, students and teachers from a public university in central Mexico. From a structural model, the results show that there is a dependence relationship between organizational climate and collaboration. Based on these findings, research lines related to trust as a determinant of knowledge networks and organizational collaboration.
\end{abstract}

\section{Keywords}

Work culture, institutionalism, leadership, knowledge network, cooperation.

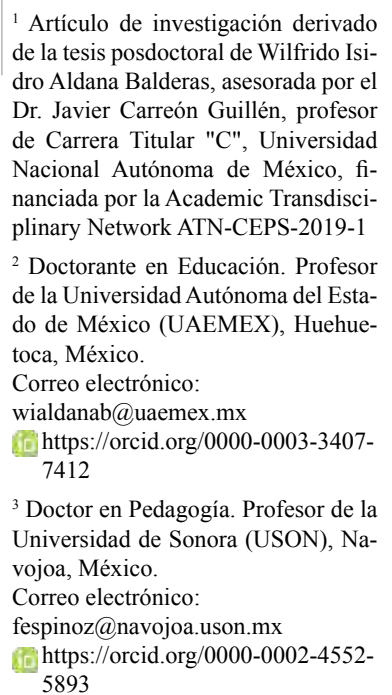

${ }^{1}$ Artículo de investigación derivado de la tesis posdoctoral de Wilfrido Isidro Aldana Balderas, asesorada por el Dr. Javier Carreón Guillén, profesor de Carrera Titular "C", Universidad Nacional Autónoma de México, financiada por la Academic Transdisciplinary Network ATN-CEPS-2019-1

${ }^{2}$ Doctorante en Educación. Profesor de la Universidad Autónoma del Estado de México (UAEMEX), Huehuetoca, México.

Correo electrónico:

wialdanab@uaemex.mx

(10 https://orcid.org/0000-0003-34077412

${ }^{3}$ Doctor en Pedagogía. Profesor de la Universidad de Sonora (USON), Navojoa, México.

Correo electrónico:

fespinoz@navojoa.uson.mx

(16) https://orcid.org/0000-0002-45525893

${ }^{4}$ Doctorante en Trabajo Social. Profesor de la Universidad Nacional Autónoma de México (UNAM), Ciudad de México, México.

Correo electrónico: jorheval@unam.mx

${ }^{5}$ Estudios de Doctorado en Psicología de la Universidad Autónoma del Estado de México (UAEMEX), Cuernavaca, México.

Correo electrónico:

garcialirios@icloud.com

[ichttps://orcid.org/0000-0002-93646796

Para citar este artículo use: AldanaBalderas, W., Espinoza-Morales, F., Hernández-Valdés, J., y GarcíaLirios, C. (2018). Especificación de un modelo para el estudio de las redes colaborativas en una universidad productora de conocimiento. Civilizar, 18(35), 91-102. doi:10.22518/usergioa/jour/ccsh/2018.2/a07 


\section{Introducción}

El objetivo del presente trabajo fue especificar un modelo para el estudio de las redes de colaboración mediante el establecimiento de la confiabilidad y la validez de un instrumento que mide el proceso organizacional en una muestra de administrativos, docentes y estudiantes de una Institución de Educación Superior del Estado de México afiliada a la Asociación Nacional de Facultades y Escuelas de Contaduría y Administración (ANFECA).

En el contexto de las políticas educativas centradas en la vinculación entre el mercado local y los curriculums académicos, el emprendimiento social a través de redes académicas supone la formación de una identidad de género o habitus que consiste en la formación profesional de una ética de la colaboración (Cruz, Arroyo y Marmolejo, 2016). Se trata de una ética que inicia con el establecimiento de la empatía, la confianza, el compromiso, la innovación, la satisfacción y la felicidad (García, Carreón, Hernández, y Salinas, 2016).

De este modo, las redes colaborativas, derivadas del institucionalismo educativo consistente en la evaluación, la acreditación y la certificación de la calidad de los procesos y productos, evidencian las diferencias entre los géneros en función de sus capacidades, habilidades y conocimientos (Hernández y Valencia, 2016).

Por consiguiente, la formación de redes colaborativas supone la capacitación o el adiestramiento de grupos en torno a objetivos, tareas y metas específicas, pero la formación de redes colaborativas en torno a la identidad profesional supone el establecimiento de un sistema de comunicación y motivación del líder hacia los talentos o entre los empleados (Acar y Acar, 2014).

\section{Estructuras organizacionales complejas}

Las redes colaborativas se gestan en torno a clima laborales como los de relaciones, los de tareas, los de apoyos y los de innovación a fin de poder emprender o especificar un conocimiento que se traducirá en una innovación según el grado de complejidad relativo a la autorregulación, la disipación, la adaptabilidad y el dinamismo (véase Tabla 1).

En esencia, las organizaciones complejas son colaborativas. Es decir, su relación con las demandas de su entorno las orienta hacia la autorregulación o el equilibrio de sus procesos al optimizar sus recursos o innovar sus capacidades en función de la disponibilidad de oportunidades. Es el caso de las organizaciones con un clima de relaciones colaborativas enfocadas en las reglas (Mendoza, Ramírez y Atriano, 2016).

Por su parte, las organizaciones disipativas generan redes colaborativas para amplificar sus opciones solidarias o climas de apoyos en función de sus posibilidades futuras. En ese mismo sentido, la teoría de las decisiones prospectivas advierte que las organizaciones disipativas son aquellas que orientan sus redes colaborativas hacia escenarios que suponen más riesgos que beneficios si estos últimos son mínimos (Osibanjo y Adeniji, 2013).

En contraste, la teoría de la decisiones prospectivas señala que las organizaciones adaptativas son aquellas que orientan sus redes colaborativas hacia escenarios de ganancias improbables con respecto a mínimos riesgos o amenazas (Escobar, 2014).

Es así como las organizaciones dinámicas, siguiendo los planteamientos de la teoría de las decisiones prospectivas, son creadoras de innovaciones y difusiones acordes a los climas laborales. En ese sentido, la producción de un conocimiento es resultado de clima colaborativos orientados por intenciones de maximización de beneficios y reducción de imponderables (Janićijević, 2013).

Las organizaciones complejas desarrollan redes colaborativas para la optimización 
Tabla 1.

Estructuras y de climas en las organizaciones complejas

\begin{tabular}{|c|c|c|c|c|}
\hline & Reglas & Apoyo & Innovación & Metas \\
\hline $\begin{array}{c}\text { Auto- } \\
\text { regulación }\end{array}$ & $\begin{array}{l}\text { Las organizaciones } \\
\text { auto-reguladas } \\
\text { establecen normas con } \\
\text { la finalidad de alcanzar } \\
\text { el equilibrio entre las } \\
\text { demandas externas y la } \\
\text { disponibilidad de los } \\
\text { recursos internos. }\end{array}$ & $\begin{array}{l}\text { Las organizaciones } \\
\text { auto-reguladas establecen } \\
\text { formas de cooperación } \\
\text { y solidaridad ante una } \\
\text { diferencia significativa } \\
\text { entre oportunidades y } \\
\text { capacidades. }\end{array}$ & $\begin{array}{l}\text { Las organizaciones } \\
\text { auto-reguladas producen } \\
\text { conocimientos e } \\
\text { innovaciones a fin de poder } \\
\text { restablecer un balance } \\
\text { entre riegos y objetivos, } \\
\text { incertidumbre y metas. }\end{array}$ & $\begin{array}{l}\text { Las organizaciones auto- } \\
\text { reguladas determinan } \\
\text { objetivos y metas con } \\
\text { base en el equilibrio } \\
\text { de sus expectativas y } \\
\text { necesidades. }\end{array}$ \\
\hline Disipación & $\begin{array}{l}\text { Las organizaciones } \\
\text { emergentes están } \\
\text { orientadas por principios } \\
\text { de restructuración } \\
\text { en función de la } \\
\text { disponibilidad de recursos } \\
\text { más que de las demandas } \\
\text { del mercado. }\end{array}$ & $\begin{array}{l}\text { Las organizaciones } \\
\text { emergentes se relacionan } \\
\text { con nodos productores } \\
\text { de conocimientos para } \\
\text { generar estructuras } \\
\text { de oportunidades } \\
\text { en situaciones de } \\
\text { desempleo. }\end{array}$ & $\begin{array}{l}\text { Las organizaciones } \\
\text { emergentes reflejan la } \\
\text { incertidumbre de las } \\
\text { crisis económicas y sus } \\
\text { innovaciones son una } \\
\text { respuesta eficaz ante la } \\
\text { incertidumbre y los riesgos } \\
\text { de emprendimiento. }\end{array}$ & $\begin{array}{l}\text { Las organizaciones } \\
\text { emergentes determinan } \\
\text { objetivos y metas } \\
\text { conforme las demandas } \\
\text { del mercado son } \\
\text { contingentes. }\end{array}$ \\
\hline Adaptación & $\begin{array}{l}\text { Las organizaciones } \\
\text { adaptativas siguen } \\
\text { principios impredecibles } \\
\text { a partir de los cuales } \\
\text { estructuras nuevas } \\
\text { oportunidades y } \\
\text { capacidades. }\end{array}$ & $\begin{array}{l}\text { Las organizaciones } \\
\text { adaptativas subyacen de } \\
\text { la incertidumbre de los } \\
\text { mercados con la finalidad } \\
\text { de estructurar nuevos } \\
\text { saberes colectivos. }\end{array}$ & $\begin{array}{l}\text { Las organizaciones } \\
\text { adaptativas generan la } \\
\text { información conducente } \\
\text { a nuevos conocimientos e } \\
\text { innovaciones para enfrentar } \\
\text { la inestabilidad de los } \\
\text { mercados. }\end{array}$ & $\begin{array}{l}\text { Las organizaciones } \\
\text { adaptativas establecen } \\
\text { objetivos y metas a } \\
\text { partir de los riesgos } \\
\text { que conllevan el } \\
\text { emprendimiento y la } \\
\text { innovación. }\end{array}$ \\
\hline Dinámica & $\begin{array}{l}\text { Las organizaciones } \\
\text { dinámicas son establece } \\
\text { inestables ante el } \\
\text { desarrollo de sus proceso } \\
\text { de calidad. }\end{array}$ & $\begin{array}{l}\text { Las organizaciones } \\
\text { dinámicas a través de } \\
\text { la cooperación y la } \\
\text { solidaridad establecen la } \\
\text { calidad de sus procesos y } \\
\text { productos. }\end{array}$ & $\begin{array}{l}\text { Las organizaciones } \\
\text { dinámicas son flexibles ante } \\
\text { la inestabilidad del mercado } \\
\text { y las demandas del Estado. }\end{array}$ & $\begin{array}{l}\text { Las organizaciones } \\
\text { dinámicas establecen } \\
\text { objetivos y metas en } \\
\text { función de los cambios } \\
\text { económicos, políticos y } \\
\text { sociales. }\end{array}$ \\
\hline Complejidad & $\begin{array}{l}\text { Las organizaciones } \\
\text { complejas generan } \\
\text { redes de conocimiento } \\
\text { a partir de las cuales } \\
\text { establecen desequilibrios } \\
\text { y estabilidad. }\end{array}$ & $\begin{array}{l}\text { Las organizaciones } \\
\text { complejas establecen } \\
\text { alianzas estratégicas } \\
\text { con la finalidad de } \\
\text { producir valor en cuanto } \\
\text { a oportunidades y } \\
\text { capacidades. }\end{array}$ & $\begin{array}{l}\text { Las organizaciones } \\
\text { complejas generan } \\
\text { innovaciones para el } \\
\text { posicionamiento y la } \\
\text { trasformación local. }\end{array}$ & $\begin{array}{l}\text { Las organizaciones } \\
\text { complejas establecen sus } \\
\text { objetivos y metas a partir } \\
\text { de las contingencias del } \\
\text { entorno. }\end{array}$ \\
\hline
\end{tabular}

Fuente: Elaboración propia, adaptada de Carreón (2016).

de recursos y la innovación de procesos ya que, su relación con su entorno las obliga a desarrollar protocolos de sustentabilidad centrados en la austeridad, la anticipación, el altruismo, la efectividad, la deliberación y el ahorro de recursos en función de la escasez de oportunidades o el incremento de las demandas externas (véase Tabla 2).

Es decir que las organizaciones complejas con redes colaborativas son en esencia sustentables ya que desarrollan una identidad de propensión al futuro, o bien, una identidad de optimización de los recursos más que de innovación de los procesos (Quintero, Velázquez, Sales y Padilla, 2016).

La orientación hacia la sustentabilidad o la afinidad hacia la conservación de los recursos distingue a las organizaciones complejas con redes colaborativas centradas 
Tabla 2.

Disposiciones temporales de las organizaciones complejas

\begin{tabular}{c|c|c|c|c|c|c}
$\begin{array}{c}\text { Auto- } \\
\text { regulación }\end{array}$ & Austeridad & Anticipación & Altruismo & Efectividad & Deliberación & Ahorro \\
\hline Disipación & $\sqrt{ }$ & $\sqrt{ }$ & & $\sqrt{ }$ & $\sqrt{ }$ & $\sqrt{ }$ \\
\hline Adaptación & $\sqrt{ }$ & & $\sqrt{ }$ & $\sqrt{ }$ & $\sqrt{ }$ & $\sqrt{ }$ \\
\hline Dinámica & & $\sqrt{ }$ & & $\sqrt{ }$ & $\sqrt{ }$ & \\
\hline Complejidad & $\sqrt{ }$ & $\sqrt{ }$ & $\sqrt{ }$ & $\sqrt{ }$ & $\sqrt{ }$ & $\sqrt{ }$
\end{tabular}

Fuente: Elaboración propia, adaptada de Carreón (2016).

en la optimización de los recursos (Sales, Quintero y Velázquez, 2016). La propensión al futuro no solo implica que las organizaciones asumen a los recursos como bienes comunes sino, además genera un proceso isomórfico en el que se circunscriben para garantizar su preservación mediante la colaboración (Robles, Alviter, Ortega y Martínez, 2016).

En ese tenor de orientación hacia la sustentabilidad, las organizaciones se estructuran en redes, neurón, grafos, nodos o arcos para establecer relaciones de cooperación, solidarias o colaborativas en función de las contingencias del entorno (Vázquez, Barrientos, Quintero y Velázquez, 2016).

Más precisamente, las organizaciones se edifican a partir de valores y normas que determinarán el procesamiento de información y con base en ello tomarán decisiones y acciones en relación con su entorno cada vez más escaso en cuanto a recursos y oportunidades. Por consiguiente, las organizaciones con orientación a la sustentabilidad deben optimizar sus recursos, generando su propias oportunidades de propensión al futuro común (Saansongu y Ngutor, 2012).

\section{Redes académicas colaborativas}

En el ámbito de las Instituciones de Educación Superior (IES), las redes colaborativas han emergido desde la psicología cognoscitiva, principalmente abordadas desde la teoría de la adopción de la tecnología, la autoeficacia y el riesgo en general y de la autoeficacia computacional y el riesgo informativo en particular (Dlugoborskyte y Petraite, 2017).

En cada una de estas tres aproximaciones, las redes colaborativas son producto de las habilidades individuales de enseñanza y aprendizaje. Incluso, las perspectivas teóricas aluden al autodidactismo, soslayando las relaciones de poder e influencia planteadas por la psicología social (Štorga, Mostashari y Stanković, 2013).

De esta manera, ante las habilidades individuales emergen experimentos que demostrarán fehacientemente la influencia social de grupos colaborativas en la retención, el aprovechamiento y el desempeño de individuos adscritos a grupos colaborativos en el marco de climas laborales centrados en las relaciones de tareas, innovaciones y apoyos (Chen, Liu y Oderanti, 2017).

Simpson (2008) revisó la literatura en torno al aprendizaje colaborativo a distancia para advertir una prevalencia de la influencia de grupo frente al aprendizaje en sus modalidades dirigidas o autodidactas. La diferencia radica en que los grupos colaborativos fortalecieron más su capacidad de retención que aquellos individuos formados desde la motivación de sus habilidades o la automotivación de sus capacidades. 
Es decir, mientras que la transferencia de conocimiento se realizaba con base en el aprovechamiento y procesamiento de información que el docente debía corregir y ajustar a una línea de aprendizaje, en los grupos colaborativos se establecían relaciones empáticas y de sinergia que potencializaba sus capacidades y habilidades de retención, aprendizaje, aprovechamiento y desempeño.

Sin embargo, ambas rutas de aprendizaje, la de competencias individuales respecto a la influencia social, oscilan entre un conocimiento tácito y un conocimiento implícito depositado en los talentos y líderes que la organización debe retener para no perder ese capital intelectual y además hacer concesiones a estos portadores del conocimiento a fin de que puedan transferir sus competencias o estrategias en futuras generaciones de educadores y estudiantes (García, Quintero y Bautista, 2017).

Es así como surge la gestión del conocimiento para resguardar la acumulación de información que solo los talentos y líderes pueden capitalizar a favor de la organización. En ese sentido, las Tecnologías de Infor- mación y Comunicación (TIC), dispositivos electrónicos y redes digitales se tornan relevantes en la construcción de activos intangibles y ventajas competitivas para las organizaciones dedicadas a la creación del conocimiento, principalmente aquellas que lo producen como es el caso de las IES en general y las universidades públicas en particular (Wu, Tuo y Xiong, 2015).

\section{Gestión de las redes de conocimiento}

La gestión del conocimiento, en un sentido computacional, supone la codificación del conocimiento implícito a un conocimiento tácito como sería el caso de las decisiones y acciones en función de la iteración de oportunidades y retos (Jabar, Sidi y Selamar, 2010).

En torno a la gestión del conocimiento, los modelos giran alrededor de las capacidades, las competencias y la habilidades individuales, por consiguiente, la mayoría de ellas en el orden implícito respecto a los modelos más tácitos y por consiguiente más empáticos, comunicativos y tácitos como es el caso del modelo sinérgico de las redes de conocimiento (véase Figura 1).

Figura 1. Modelo sinérgico del conocimiento

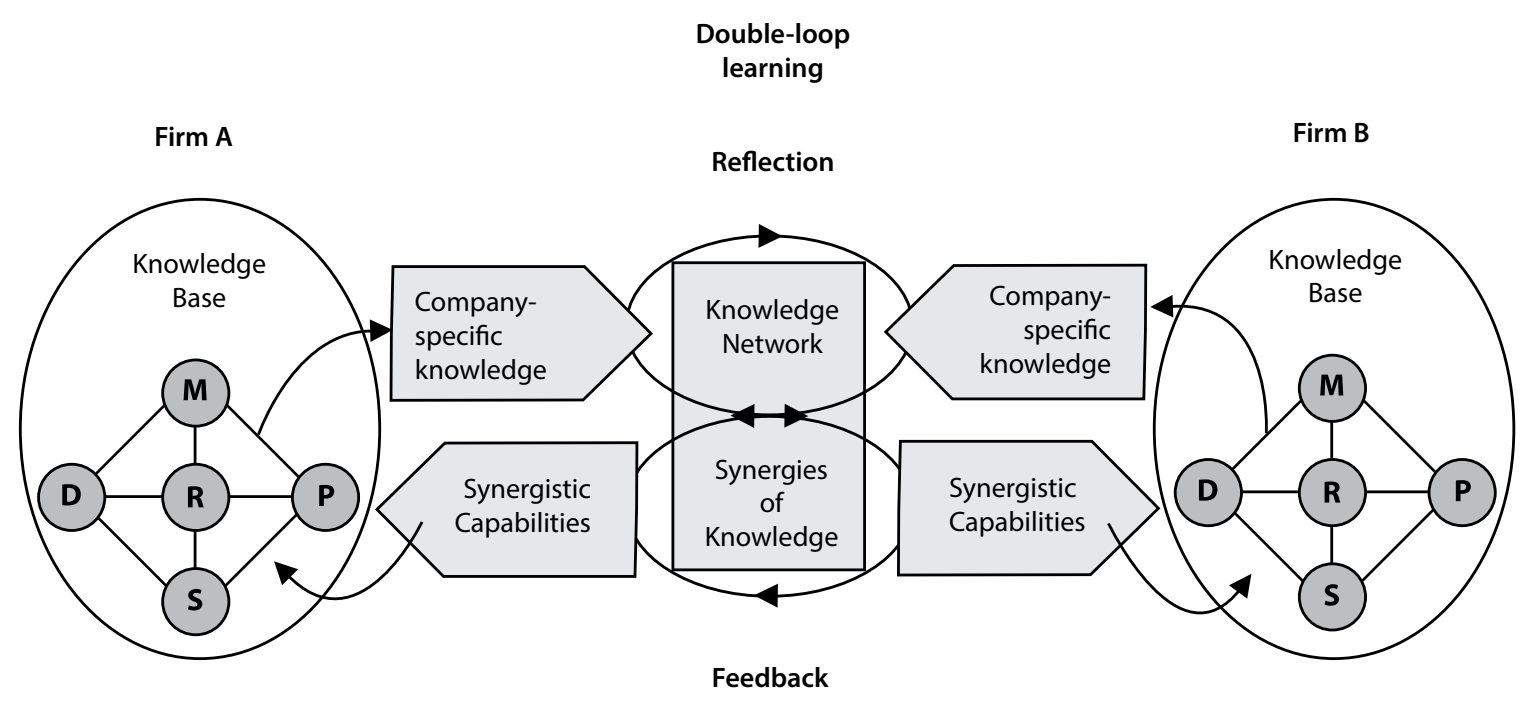

Fuente: Elaboración propia, adaptado de Nielsen (2002). 
De este modo, entre el modelo sinérgico de influencia social mediada por alguna tecnología y el modelo de competencias y trasferencia vertical, grosso modo, el modelo intermedio de gestión del conocimiento o conversión y resguardo de la información producto de la experiencia y las habilidades individuales incluye tres fases: 1) procesamiento de captura de conocimiento a través del mapeo conceptual de la información de agentes del conocimiento y agentes receptores; 2) construcción y administración de perfiles de usuarios, competencias y redes de conocimiento; 3 ) generación de estructuras de conocimiento para el repositorio de conocimiento grupal (García, 2013).

Sin embargo, en este enfoque de la gestión del conocimiento, prevalece una comunicación y motivación unidireccional y por tanto unilateral. Es decir, el conocimiento es gestionado, producido y transferido en un sentido vertical sin considerar las necesidades de los usuarios ni sus posibilidades de interacción de expectativas de utilidad.

Por consiguiente, es menester profundizar en el interior de una red de conocimiento académica con sentido colaborativo, el cual podrá observarse en sus niveles de relaciones, innovaciones, apoyos y metas acordes a las contingencias de su entorno como es el caso de un sistema de prácticas y servicio social implementado entre la universidad pública y sucursales de una multinacional automotriz del centro de México.

En el contexto de la alianza estratégica entre la IES y la multinacional, el sistema de prácticas y servicio social emerge como un escenario para el estudio de las redes colaborativas derivadas de la gestión, producción y transferencia del conocimiento, pero debido a que tal alianza se orienta hacia la inserción laboral del estudiante, por consiguiente, será menester observar sus dimensiones de apoyos, innovaciones, metas y relaciones.

\section{Formulación}

¿Las dimensiones organizacionales con orientación a la complejidad de sus procesos colaborativos y sustentables esgrimidas en la literatura consultada se ajustarán a las observaciones empíricas a realizar en un estudio de caso con estudiantes, administrativos y docentes de una universidad pública del centro de México?

\section{Hipótesis nula}

Las organizaciones dedicadas a la creación de conocimiento mediante redes colaborativas desarrollan y consolidan dimensiones de complejidad relativas a la auto-regulación, la disipación, la adaptabilidad y el dinamismo como sello distintivo de la optimización de recursos y la innovación de sus procesos. De esta forma, será posible observar tales dimensiones en un estudio de caso con una IES del centro de México.

\section{Hipótesis alterna}

No obstante que las teorías y hallazgos empíricos revisados en la literatura advierten que las organizaciones desarrollan redes colaborativas complejas con la finalidad de auto-regularse, disiparse, adaptarse y dinamizarse ante la escasez de los recursos, las especificidades del contexto y las particularidades de la muestra de estudio advierten que se trata de un fenómeno inédito y por tanto explorable en cuanto a sus estructuras organizaciones.

\section{Método}

Se llevó a cabo un estudio no experimental, trasversal y exploratorio. Se realizó una selección no probabilística de 300 administrativos, docentes y estudiantes de una universidad pública, afiliada a la ANFECA del área cinco.

Se utilizó la Escala de Colaboración Organizacional de Carreón (2016) la cual 
incluye cuatro dimensiones relativas al clima de relaciones, apoyos, innovaciones y metas. Cada ítem se responde con alguna de cinco opciones que van desde "nada de acuerdo" hasta muy de acuerdo".

Se utilizó la técnica Delphi para la homogeneización de las palabras incluidas en las aseveraciones del instrumento. Se garantizó por escrito la confidencialidad de los resultados y se informó acerca de que estos no afectarían ni negativa ni positivamente su estatus laboral-administrativo. La información fue procesada en el Paquete Estadístico para Ciencias Sociales (SPSS por sus siglas en inglés). Se estimaron media, desviación estándar, alfa, esfericidad, adecuación, pesos factoriales, bondad de ajuste y residuales.

\section{Resultados}

La Tabla 3 muestra las propiedades estadísticas del instrumento que estableció cuatro dimensiones de la colaboración organizacional, a saber: el clima de relaciones, apoyos, innovaciones y metas con coeficientes alfa de Cronbach superiores al mínimo indispensable $(0,700)$ para considerarlo como una medición consistente.

Una vez establecidos los cuatro factores que explicaron el $71 \%$ de la varianza total explicada, se procedió a estimar las relaciones entre los factores con la finalidad de establecer las posibles relaciones de la estructura factorial con respecto a otras variables no especificadas ni estimadas en el modelo.

Figura 2.

Modelo estructural de relaciones entre los factores

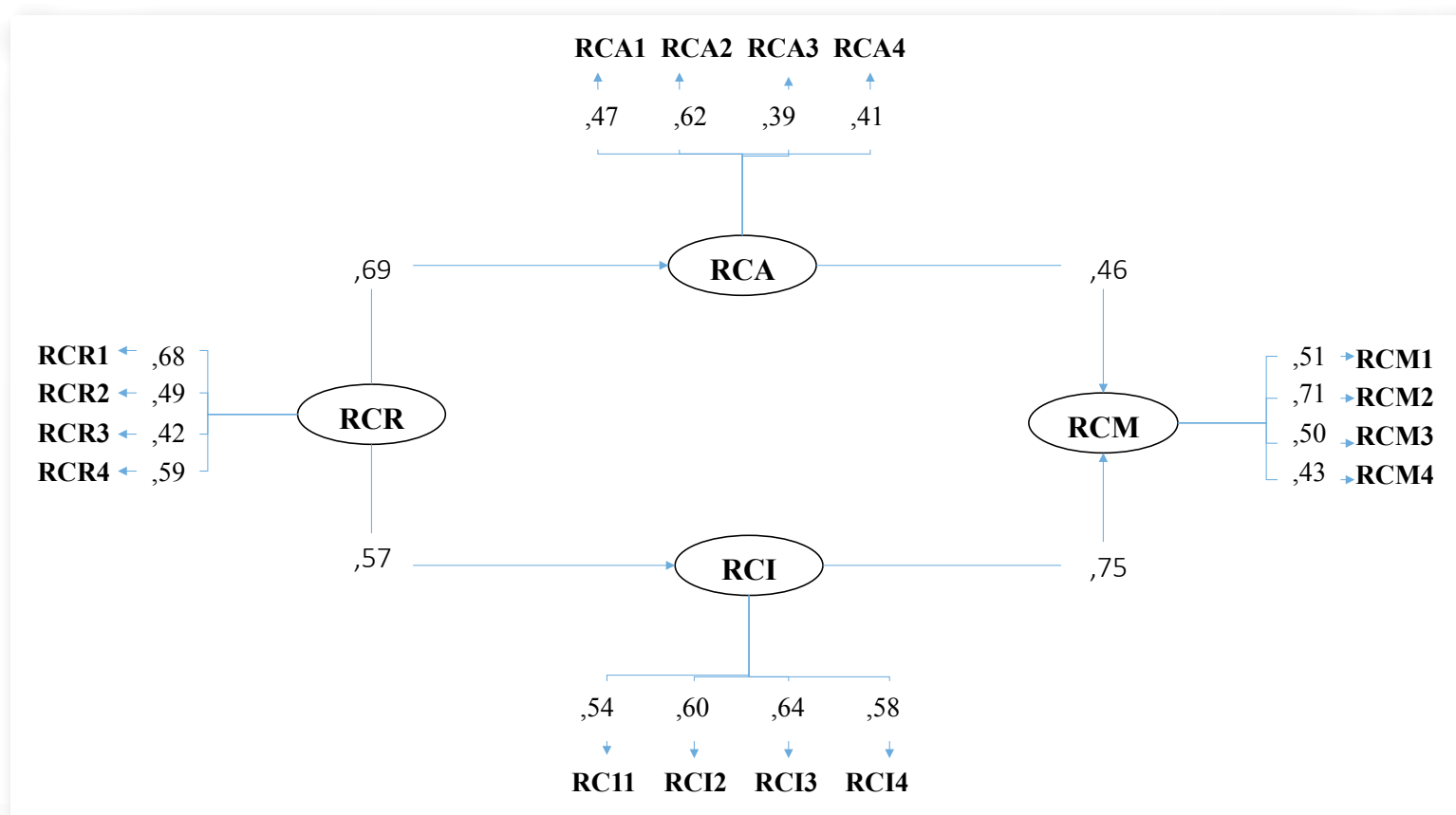

$\mathrm{RCR}=$ Redes Colaborativas de Relaciones, $\mathrm{RCA}=$ Redes Colaborativas de Apoyos, $\mathrm{RCI}=$ Redes Colaborativas de Innovaciones, $\mathrm{RCM}$ $=$ Redes Colaborativas de Metas

Fuente: Elaboración propia. 
Tabla 3.

Descriptivos del instrumento

\begin{tabular}{|c|c|c|c|c|c|c|c|c|c|c|}
\hline Clave & Ítem & M & D & S & C & $\mathbf{A}$ & F1 & F2 & F3 & F4 \\
\hline & $\begin{array}{c}\text { Subescala de relaciones } \\
\text { (especificaciones ante generalidades) }\end{array}$ & & & & & 0,781 & & & & \\
\hline RS1 & $\begin{array}{l}\text { La evaluación educativa genera artículos } \\
\text { indizados }\end{array}$ & 3,24 & 1,25 & 1,02 & 1,32 & 0,743 & 0,632 & & & \\
\hline RS2 & $\begin{array}{l}\text { La producción de innovaciones es acorde } \\
\text { a la calidad educativa }\end{array}$ & 3,29 & 1,27 & 1,39 & 1,35 & 0,793 & 0,694 & & & \\
\hline RS3 & $\begin{array}{l}\text { La asesoría de tesis deriva del concurso } \\
\text { de méritos }\end{array}$ & 3,00 & 1,47 & 1,40 & 1,38 & 0,714 & 0,661 & & & \\
\hline \multirow[t]{2}{*}{ RS4 } & $\begin{array}{l}\text { Los proyectos de investigación surgen del } \\
\text { credencialismo }\end{array}$ & 4,28 & 1,85 & 1,44 & 1,54 & 0,756 & 0,632 & & & \\
\hline & $\begin{array}{l}\text { Subescala de apoyos (colaboraciones } \\
\text { ante imponderables) }\end{array}$ & & & & & 0,793 & & & & \\
\hline AP1 & $\begin{array}{l}\text { La educación de masas supuso el } \\
\text { debilitamiento del sindicalismo }\end{array}$ & 3,05 & 1,04 & 1,05 & 1,47 & 0,742 & & 0,631 & & \\
\hline AP2 & $\begin{array}{l}\text { El individualismo docente obedece a las } \\
\text { políticas educativas }\end{array}$ & 3,81 & 1,37 & 1,28 & 1,39 & 0,746 & & 0,635 & & \\
\hline AP3 & $\begin{array}{l}\text { El neoliberalismo educativo genera los } \\
\text { trabajos colegiados }\end{array}$ & 3,21 & 1,21 & 1,38 & 1,27 & 0,784 & & 0,563 & & \\
\hline \multirow[t]{2}{*}{ AP4 } & $\begin{array}{l}\text { La investigación multidisciplinar es } \\
\text { producto de la meritocracia }\end{array}$ & 3,56 & 2,31 & 1,29 & 1,07 & 0,795 & & 0,594 & & \\
\hline & $\begin{array}{l}\text { Subescala de innovaciones (propuestas } \\
\text { ante contingencias) }\end{array}$ & & & & & 0,785 & & & & \\
\hline IN1 & $\begin{array}{l}\text { Las propuestas docentes subyacen de la } \\
\text { crisis educativa }\end{array}$ & 4,21 & 1,70 & 1,33 & 1,21 & 0,790 & & & 0,671 & \\
\hline IN2 & $\begin{array}{l}\text { La deserción educativa generó el sistema } \\
\text { de becas }\end{array}$ & 4,24 & 1,48 & 1,20 & 1,24 & 0,712 & & & 0,493 & \\
\hline IN3 & $\begin{array}{l}\text { El construccionismo es resultado del } \\
\text { rezago educativo }\end{array}$ & 3,91 & 1,31 & 1,25 & 1,36 & 0,774 & & & 0,614 & \\
\hline \multirow[t]{2}{*}{ IN4 } & $\begin{array}{l}\text { Las políticas educativas propiciaron el } \\
\text { credencialismo }\end{array}$ & 3,26 & 1,83 & 1,37 & 1,32 & 0,732 & & & 0,632 & \\
\hline & Subescala de metas (logros ante riesgos) & & & & & 0,758 & & & & \\
\hline MT1 & $\begin{array}{l}\text { El recorte presupuestal generó el } \\
\text { ausentismo }\end{array}$ & 4,34 & 1,83 & 1,09 & 1,30 & 0,795 & & & & 0,381 \\
\hline MY2 & $\begin{array}{l}\text { Los reconocimientos son derivados de la } \\
\text { educación de masas }\end{array}$ & 4,65 & 1,57 & 1,15 & 1,26 & 0,782 & & & & 0,532 \\
\hline MT3 & $\begin{array}{l}\text { Los estudios multidisciplinares indican la } \\
\text { política tecnocientífica }\end{array}$ & 4,81 & 1,46 & 1,13 & 1,32 & 0,784 & & & & 0,635 \\
\hline MT4 & $\begin{array}{l}\text { Los financiamientos educativos se } \\
\text { consiguen con la gestión }\end{array}$ & 4,30 & 1,24 & 1,36 & 1,49 & 0,793 & & & & 0,512 \\
\hline
\end{tabular}

$\mathrm{M}=$ Media, $\mathrm{D}=$ Desviación estándar, $\mathrm{S}=$ Sesgo, $\mathrm{C}=$ Curtosis, $\mathrm{A}=$ Alfa quitando el valor del ítem. Método de extracción: Componentes principales. Esfericidad y adecuación $\left\lceil\chi^{2}=3,251(23 \mathrm{gl}) \mathrm{p}=0,000 ; \mathrm{KMO}=0,681\right\}$. F1 $=$ Clima de Relaciones $(45 \%$ de la varianza total explicada), F2 = Clima de Apoyos ( $15 \%$ de la varianza total explicada), F3 = Clima de Innovaciones ( $8 \%$ de la varianza total explicada), F3 = Clima de metas (3\% de la varianza total explicada). Todos los ítems incluyen el valor alfa quitando su estimación e incluyen cinco opciones de respuesta: 0 = "nada de acuerdo" hasta 5 = "Muy de acuerdo".

Fuente: Elaboración propia. 
Tabla 4.

Relaciones entre los factores y el constructo

\begin{tabular}{l|c|c|c|c|c|c} 
& & Estimación & S.E. & C.R. & P \\
\hline Relaciones & $\leftarrow$ & Colaboración & 0,50 & & & \\
\hline Apoyos & $\leftarrow$ & Colaboración & $-0,10$ &, 586 &, 837 &, 403 \\
\hline Innovaciones & $\leftarrow$ & Colaboración & $-0,17$ & 2,679 &, 911 &, 362 \\
\hline Metas & $\leftarrow$ & Colaboración & $-0,33$ &, 667 & $-1,341$ &, 180
\end{tabular}

Fuente: Elaboración propia.

Una vez establecidas las relaciones entre los factores, se procedió a estimar el modelo estructural (véase Figura 2) en el que los parámetros de ajuste y residual $\lceil\chi 2=3,432(2 \mathrm{gl}) \mathrm{p}=$ 0,$180 ;$ GFI $=0,950 ;$ RMSEA $=0,001$; Bootsrap $=0,0000 \mathrm{~J}$ sugieren la aceptación de la hipótesis nula relativa al ajuste de las explicaciones teóricas respecto a las observaciones empíricas realizadas en el estudio de caso de la universidad pública.

\section{Discusión}

El aporte del presente trabajo al estado de la cuestión radica en la especificación de un modelo para el estudio de las organizaciones complejas con redes colaborativas orientadas hacia la sustentabilidad, pero el tipo de estudio no experimental, el tipo de muestreo no probabilístico y el tipo de análisis factorial exploratorio limitan los hallazgos a la muestra de estudio.

En relación con los estudios de las redes colaborativas los cuales destacan el isomorfismo y la propensión al futuro, el presente trabajo ha demostrado que prevalecen cuatro dimensiones relativas a las redes colaborativas de relaciones, apoyos, innovaciones y metas, pero el porcentaje de la varianza total explicada supone la inclusión de otros factores que la literatura identifica como clima de tareas y redes de confianza.

Es decir que las redes colaborativas distinguen a las organizaciones complejas ante las contingencias de su entorno, pero son las normas y los valores los que destacan como indicadores de las organizaciones dedicadas a la producción del conocimiento y la formación de capital intelectual con la finalidad de crear valor intangible en sus procesos.

De este modo el presente trabajo ha evidenciado la formación de redes colaborativas desde el clima de relaciones hasta el clima de metas lo cual sugiere que: 1) en las organizaciones complejas enfocadas en la auto-regulación las redes colaborativas edifican objetivos, tareas y metas con base en el equilibrio de las demandas externas y sus recursos internos; 2) en las organizaciones sustentables las redes colaborativas fungen como disipadoras de conocimientos para incrementar la formación del capital intelectual; 3) en las organizaciones productoras de conocimiento la adaptabilidad es una instancia que vendrá a generar más conocimientos a fin de conservar a la propia organización y sus recursos; 4) las organizaciones que optimizan sus recursos forman redes colaborativas para activar una dinámica de intercambios y tran sacciones con su entorno contingente.

Por tanto, es necesario llevar a cabo el contraste del modelo especificado en contextos y muestras similares a la IES de estudio, así como la inclusión de un quinto factor para incrementar el porcentaje de varianza explicada y orientar el modelo hacia la formación de capital intelectual dedicado a la optimización de recursos y a la innovación de procesos.

De esta manera, la inclusión del clima de relaciones empáticas o de confianza en el 
modelo permitirá observar su vinculación con la formación de redes académicas y profesionales en un sistema de prácticas profesionales en el marco de las alianzas estratégicas entre IES y multinacionales.

Es decir que, la construcción de una ética de la preservación y la colaboración o clima de relaciones empáticas y de confianza entre estudiantes, docentes y administrativos supone el inicio de un proceso selectivo de información que al ser decodificado permitirá resguardar el conocimiento implícito en alguna tecnología con la finalidad de potenciar las capacidades, competencias y habilidades de futuros usuarios ante escenarios cada vez más riesgosos, contingentes y amenazantes.

Esto es así porque la transferencia del conocimiento implícito en un conocimiento tácito se gesta desde el clima laboral centrado en la empatía y la confianza más que en la competencia. Por consiguiente, un modelo de gestión del conocimiento implica la inclusión y la medición del nivel de confianza y empatía de una organización, así como de sus competencias en función de las demandas del entorno y las oportunidades del contexto.

Tal modelo incluiría: 1) gestión del conocimiento a partir de un diagnóstico del nivel de empatía y confianza; 2) producción del conocimiento con base en los objetivos, tareas y metas comunes entre IES y multinacionales; 3 ) transferencia del conocimiento a través del sistema de prácticas y servicio social, así como la formación académica, tecnológica y profesional de activos intangibles como el capital intelectual.

\section{Conclusión}

El objetivo del presente trabajo ha sido especificar un modelo para el estudio de las organizaciones complejas con redes colaborativas que optimicen los recursos e innoven los procesos frente a las contingencias de su entorno, pero el tipo de estudio, muestra y análisis limita el modelo a la muestra de estudio, aunque sugiere su contraste en otros escenarios. Se trata de un modelo a partir del cual será posible conformar grupos colaborativas con base en la edificación de una identidad o propensión al futuro compartido.

\section{Referencias}

Acar, Z., y Acar, P. (2014). Organizational culture types and their effects on organizational performance in Turkish hospitals. Emerging Markets Journal, 3(3), 18-31. doi: 10.5195/emaj.2014.47

Carreón, J. (2016). Desarrollo humano: Gobernanza y Emprendimiento social. México: UNAM-ENTS.

Cruz, O., Arroyo, P., y Marmolejo, J. (2016). Innovaciones tecnológicas en la logística: gestión de inventarios, sistemas de información y tercerización de operaciones. En M. Quintero, J. Sales, y E. Velázquez (Coords.). Innovación y tecnología. Retos para su aplicación práctica en las empresas (pp. 165-178). México: Miguel Ángel Porrúa-Uaemex.

Chen, H., Liu, S., y Oderanti, F. (2017). A Knowledge Network and Mobilisation Framework for Lean Supply Chain Decisions in Agri-Food Industry. International Journal of Decision Support System Technology (IJDSST), 9(4), 37-48. doi:10.4018/IJDSST.2017100103

Dlugoborskyte, V., y Petraite, M. (2017). Framework for explaining the formation of knowledge intensive entrepreneurial born global firm: Entrepreneurial, strategic and network based constituents. Journal of Evolutionary Studies in Business, 2(1), 174-202. doi: 10.1344/jesb2017.1.j026

Escobar, R. (2014). Redes neuronales, procesos cognoscitivos y análisis de la conducta. Revista Internacional de Conductismo, 2(1), 23-43. 
García, C. (2013). La red de conocimiento en una universidad con sistema de prácticas profesionales y servicio social tecnológico administrativo. Fundamentos en $\mathrm{Hu}$ manidades, 14(27), 135-157

García, C., Carreón, J., Hernández, J y Salinas, R. (2016). Gobernanza de los actores y redes de innovación tecnológica. En M. Quintero, J. Sales, y E. Velázquez (Coords.). Innovación y tecnología. Retos para su aplicación práctica en las empresas (pp. 79-94). México: Miguel Ángel Porrúa-Uaemex.

García, C., Quintero, M. L., y Bautista, M. (2017). Redes de conocimiento en torno a la caficultura en una IES del centro de México. Interconectando Saberes, 2(4), $15-32$

Hernández, A., y Valencia, R. (2016). Instrumentos de innovación: las redes sociales en la internalización de las micro, pequeñas y medianas empresas mexicanas. En M. Quintero, J. Sales, y E. Velázquez (Coords.). Innovación y tecnología. Retos para su aplicación práctica en las empresas (pp. 47-66). México: Miguel Ángel Porrúa-Uaemex.

Jabar, M., Sidi, F., y Selamar, H. (2010). Tacit knowledge codification. Journal of Computer Science, 6(10), 1170-1176. doi:10.3844/jessp.2010.1170.1176

Janićijević, N. (2013). The mutual impact of organizational culture and structure. Economic Annals, 58(198), 35-60. doi: 10.2298/ EKA1398035J

Mendoza, E., Ramírez, L., y Atriano, R. (2016). Uso de los medios y las tecnologías en la creación de un sistema de innovación para el bien común. En M. Quintero, J. Sales, y E. Velázquez (Coords.). Innovación y tecnología. Retos para su aplicación práctica en las empresas (pp. 95-114). México: Miguel Ángel Porrúa-Uaemex.
Nielsen, B. (2002). Synergies in strategic alliances: motivation and outcomes of complementary and synergistic knowledge networks. Journal of Knowledge Management Practice, 3(2), 1-15.

Osibanjo, A. O. y Adeniji, A. A. (2013) Impact of Organizational Culture on Human Resource Practices: A Study of Selected Nigerian Private Universities. Journal of Competitiveness, 5(4). 115-133. doi:10.7441/joc.2013.04.07

Quintero, M., Velázquez, E., Sales, J. y Padi1la, S. (2016). Una revisión del estado del arte sobre pymes. ¿y los estudios de innovación? En M. Quintero, J. Sales, y E. Velázquez (Coords.). Innovación y tecnología. Retos para su aplicación práctica en las empresas (pp. 31-43). México: Miguel Ángel Porrúa-Uaemex.

Robles, C., Alviter, L., Ortega, A., y Martínez, E. (2016). Cultura de calidad e innovación en la microempresa. En M. Quintero, J. Sales, y E. Velázquez (Coords.). Innovación y tecnología. Retos para su aplicación práctica en las empresas (pp. 11-30). México: Miguel Ángel PorrúaUaemex.

Saansongu, E., y Ngutor, D. (2012). The influence of corporate culture of employee commitment to the organization. International Journal of Business and Management, 7(22), 21-28. doi:10.5539/ijbm. $\mathrm{v} 7 \mathrm{n} 22 \mathrm{p} 21$

Sales, J., Quintero, M., y Velázquez, E. (2016). Adaptación versus innovación: la formación de distritos industriales a partir de comunidades campesinas. Santa Cruz Atizapan y Chiconcuac. En M. Quintero, J. Sales, y E. Velázquez (Coords.). Innovación y tecnología. Retos para su aplicación práctica en las empresas (pp. 181-199). México: Miguel Ángel Porrúa-Uaemex. 
Simpson, O. (2008). Motivating learners in open and distance learning: Do we need a new theory of learner support? Open learning, 23(3), 159-170. doi:10.1080/02680510802419979

Štorga, M., Mostashari, A., y Stanković, T. (2013). Visualisation of the organisation knowledge structure evolution. Journal of Knowledge Management, 17(5), 724740. doi:10.1108/JKM-02-2013-0058

Vázquez, C., Barrientos, B., Quintero, M., y Velázquez, E. (2016). Apoyos gubernamentales para la innovación, tecnología y capacitación de las pequeñas y medianas empresas en México. En M. Quintero, J. Sales, y E. Velázquez (Coords.). Innovación y tecnología. Retos para su aplicación práctica en las empresas (pp. 67-78). México: Miguel Ángel Porrúa-Uaemex.
Wu, S., Tuo, M., y Xiong, D. (2015). Network structure detection and analysis of Shanghai stock market. Journal of Industrial Engineering and Management, 8(2), 383398. doi: 10.3916/jiem. 1314 